DESY 88-013

February 1988

CALCULATION OF TRANSVERSE AND LONGITUDINAL SPACE CHARGE EFFECTS WITHIN THE FRAMEWORK OF THE FULLY SIX-DIMENSIONAL FORMALISM

by

I. Borchardt, E. Karantzoulis, H. Mais, G. Ripken Deutsches Elektronen-Synchrotron DESY, Hamburg 


\title{
Calculation of Transverse and Longitudinal Space Charge Effects within the Framework of the fully six-dimensional Formalism
}

\author{
I. Borchardt, E. Karantzoulis, H. Mais, G. Ripken
}

December, 1987

\begin{abstract}
In the following report we describe a method for calculating the envelope of a particle bunch in linear coupled storage rings and transport systems in the presence of transverse and longitudinal space charge forces using the (canonical) variables $x, p_{x}, z, p_{z}, \sigma=$ $s-v_{0} \cdot t, p_{\sigma}=\Delta E / E_{0}$ of the fully six-dimensional formalism. This work is an extension of earlier calculations on transverse space charge forces [1] to include the synchrotron oscillations. The extension is achieved by defining a 6 -dimensional ellipsoid in the $x-$ $p_{x}-z-p_{z}-\sigma-p_{\sigma}$ space. The motion of this ellipsoid under the influence of the external fields and the instantaneous space charge forces can be described by six generating orbit vectors which can be combined into a.6-dimensional matrix $\underline{B}(s)$. This "bunch -shape matrix", $\underline{B}(s)$, contains complete information about the configuration of the bunch. The solution of the equations of motion is carried through in the thin lens approximation. The formalism can also encompass acceleration by cavity fields.
\end{abstract}

\section{Contents}

1 Introduction $\quad 2$

2 The Equations of Motion $\quad 2$

3 The Beam Envelopes $\quad 6$

3.1 The six dimensional ellipsoid in $x-p_{x}-z-p_{z}-\sigma-p_{\sigma}$ space . . . . . . 6

3.2 The projections of the six dimensional ellipsoid . . . . . . . . . . 6

3.2.1 Projection on the $x-z$ plane. . . . . . . . . . . . 7

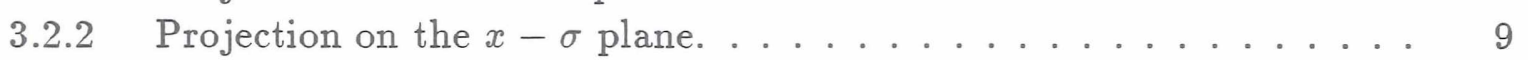

3.2.3 Projection on the $z-\sigma$ plane. ............... 10

4 Calculation of Space Charge Forces $\quad 11$

5 Solution of the Equations of Motion $\quad 14$

$\begin{array}{llr}6 & \text { Summary } & 16\end{array}$

$\begin{array}{ll}\text { Acknowledgements } & 16\end{array}$ 


\section{Introduction}

In DESY report 87-161 we described a technique for calculating the beam envelope in a storage ring when transverse space charge forces are taken into account [1]. The method consists of calculating the motion of the 5 -dimensional ellipsoid in $x-p_{x}-z-p_{z}-\Delta p / p$ space. The beam envelope at each place in the ring is obtained by projecting this ellipsoid on the $x-z, x-p_{x}$ and $z-p_{z}$ planes.

In that paper we dealt only with transverse (betatron) oscillations. In this paper we include the longitudinal space charge forces and the general case of coupled synchro-betatron motion using the 6-dimensional canonical formalism of particles with arbitrary average speed $v_{0}$ that was developed in Desy report 87-036 [2].

\section{The Equations of Motion}

The investigation of synchro-betatron oscillations in the presence of space charge forces begins with a statement of the equations of motion. We will use the same variables as those in Ref. 2: $x, z, \sigma=s-v_{0} \cdot t$ and $\eta=\Delta E / E_{0}$, where $x$ and $z$ describe the amplitude of transverse motion (betatron oscillations), while $\sigma$ and $\eta$ describe the longitudinal (synchrotron) oscillations. The quantity $\sigma$ defines the longitudinal separation of particles from the centre of the bunch.

The equations for transverse motion have already been given in Ref. 1. They are :

$$
\begin{aligned}
& x^{\prime \prime}=-G_{1} \cdot x+\left(N+H^{\prime}\right) \cdot z+2 H \cdot z^{\prime}+\frac{1}{\gamma_{0} \cdot m_{0} \cdot v_{0}^{2}} \cdot F_{x}^{s e l f}+K_{x} \cdot f(\eta) ; \\
& z^{\prime \prime}=-G_{2} \cdot z+\left(N-H^{\prime}\right) \cdot x-2 H \cdot x^{\prime}+\frac{1}{\gamma_{0} \cdot m_{0} \cdot v_{0}^{2}} \cdot F_{z}^{s e l f}+K_{z} \cdot f(\eta)
\end{aligned}
$$

with

$$
\begin{aligned}
N & =\frac{1}{2} \cdot \frac{e}{p_{0} \cdot c} \cdot\left(\frac{\partial B_{x}}{\partial x}-\frac{\partial B_{z}}{\partial z}\right)_{x=z=0} ; \\
H & =\frac{1}{2} \cdot \frac{e}{p_{0} \cdot c} \cdot B_{s} \\
g & =\frac{e}{p_{0} \cdot c} \cdot\left(\frac{\partial B_{z}}{\partial x}\right)_{x=z=0}
\end{aligned}
$$

and

$$
\begin{aligned}
G_{1} & =K_{x}^{2}+g ; G_{2}=K_{z}^{2}-g ; \\
f(\eta) & =\frac{p-p_{0}}{p_{0}}=\frac{\Delta p}{p_{0}}=\frac{1}{\beta_{0}} \cdot \sqrt{(1+\eta)^{2}-\left(m_{0} c^{2} / E_{0}\right)^{2}}-1
\end{aligned}
$$


and where $F_{x}^{\text {self }}$ and $F_{z}^{\text {self }}$ are the space charge forces in the $\mathrm{x}$ and $\mathrm{z}$ directions.

In the following we put

$$
\begin{aligned}
& \frac{1}{\gamma_{0} \cdot m_{0} \cdot v_{0}^{2}} \cdot F_{x}^{\text {self }}=F_{x x} \cdot x+F_{x z} \cdot z ; \\
& \frac{1}{\gamma_{0} \cdot m_{0} \cdot v_{0}^{2}} \cdot F_{z}^{\text {self }}=F_{z x} \cdot x+F_{z z} \cdot z
\end{aligned}
$$

where $F_{x x}, F_{x z}, F_{z x}, F_{z z}$ are introduced in chapter 4. For now, we only need to use the fact that

$$
F_{x z}=F_{z x} \text {. }
$$

The quantity $f(\eta)$ in eqn. (2.1) can be developed in a power series in $\eta$ :

$$
f(\eta)=f^{\prime}(0) \cdot \eta+\frac{1}{2} \cdot f^{\prime \prime}(0) \cdot \eta^{2}+\ldots
$$

with

$$
\begin{aligned}
f^{\prime}(\eta) & =\frac{1}{\beta_{0}} \cdot \frac{1+\eta}{\sqrt{(1+\eta)^{2}-\left(m_{0} c^{2} / E_{0}\right)^{2}}}=\frac{1}{\beta_{0}} \cdot \frac{E}{p \cdot c}=\frac{1}{\beta_{0} \cdot \beta} \\
\Rightarrow & f^{\prime}(0)=\frac{1}{\beta_{0}^{2}} ; \\
f^{\prime \prime}(\eta)= & \frac{1}{\beta_{0}} \cdot\left[(1+\eta)^{2}-\left(\frac{m_{0} c^{2}}{E_{0}}\right)^{2}\right]^{-1 / 2} \\
& -\frac{1}{\beta_{0}} \cdot(1+\eta)^{2} \cdot\left[(1+\eta)^{2}-\left(\frac{m_{0} c^{2}}{E_{0}}\right)^{2}\right]^{-3 / 2} \\
& \Rightarrow-\frac{1}{\beta_{0}} \cdot \frac{E_{0}}{E} \cdot \frac{1}{\beta^{3} \gamma^{2}} \\
& f^{\prime \prime}(0)=-\frac{1}{\beta_{0}^{4} \cdot \gamma_{0}^{2}} .
\end{aligned}
$$

To obtain the equations for longitudinal motion we recall that the field in a cavity can be written in terms of $\sigma$ as

$$
\begin{gathered}
\varepsilon_{\text {Cavity }}=V(s) \cdot \sin \left[k \cdot \frac{2 \pi}{L} \cdot \sigma+\varphi\right] \\
\varphi=0, \pi \text { for protons. }
\end{gathered}
$$

Writing the longitudinal space charge forces as

$$
\frac{1}{E_{0}} F_{s}^{s e l f}=F_{\sigma}(s) \cdot \sigma
$$

the equation for the variation of $\eta$ is:

$$
\eta^{\prime}=\frac{e V(s)}{E_{0}} \cdot \cos \varphi \cdot k \cdot \frac{2 \pi}{L} \cdot \sigma+F_{\sigma}(s) \cdot \sigma .
$$


The calculation of $F_{\sigma}$ is given in chapter 4.

The variation of $\sigma=s-v_{0} \cdot t(s)$ is given by:

$$
\begin{aligned}
& \sigma^{\prime}= 1-v_{0} \cdot \frac{d t}{d s} ; \\
& d t=\frac{d l}{v} ; \\
& d l=d s \cdot\left[1+K_{x} \cdot x+K_{z} \cdot z+\ldots\right] ; \\
& \Longrightarrow \sigma^{\prime}=1-\frac{v_{0}}{v} \cdot\left[1+K_{x} \cdot x+K_{z} \cdot z+\ldots\right] .
\end{aligned}
$$

With the relation

$$
\begin{aligned}
\frac{v_{0}}{v} & =\beta_{0}^{2} \cdot f^{\prime}(\eta) \\
f^{\prime}(\eta) & =f^{\prime}(0)+\eta \cdot f^{\prime \prime}(0)+\ldots \\
& =\frac{1}{\beta_{0}^{2}}-\eta \cdot \frac{1}{\beta_{0}^{4} \cdot \gamma_{0}^{2}}+\ldots
\end{aligned}
$$

from (2.8) and (2.9) we then obtain in linear approximation:

$$
\sigma^{\prime}=\frac{1}{\beta_{0}^{2} \gamma_{0}^{2}} \cdot \eta-\left(K_{x} \cdot x+K_{z} \cdot z\right)
$$

Equations (2.1), (2.12) and (2.14) provide a complete description of transverse and longitudinal motion in the presence of space charge forces.

To proceed further, it will be useful to write these equations in canonical form :

$$
\begin{aligned}
x^{\prime} & =\frac{\partial \hat{H}}{\partial p_{x}} ; p_{x}^{\prime}=-\frac{\partial \hat{H}}{\partial x} ; \\
z^{\prime} & =\frac{\partial \hat{H}}{\partial p_{z}} ; p_{z}^{\prime}=-\frac{\partial \hat{H}}{\partial z} ; \\
\sigma^{\prime} & =\frac{\partial \hat{H}}{\partial p_{\sigma}} ; p_{\sigma}^{\prime}=-\frac{\partial \hat{H}}{\partial \sigma}
\end{aligned}
$$

using the Hamiltonian

$$
\begin{aligned}
\hat{H}= & \frac{1}{\beta_{0}^{2} \gamma_{0}^{2}} \cdot \frac{1}{2} p_{\sigma}^{2}-\left(K_{x} \cdot x+K_{z} \cdot z\right) \cdot p_{\sigma} \\
& -\frac{1}{2} \cdot \frac{e V(s)}{E_{0}} \cdot k \cdot \frac{2 \pi}{L} \cdot \cos \varphi \cdot \sigma^{2}-\frac{1}{2} F_{\sigma}(s) \cdot \sigma^{2} \\
& +\frac{1}{2 \beta_{0}^{2}} \cdot\left\{\left[p_{x}+\beta_{0}^{2} \cdot H \cdot z\right]^{2}+\left[p_{z}-\beta_{0}^{2} \cdot H \cdot x\right]^{2}\right\} \\
& +\frac{1}{2} \beta_{0}^{2} \cdot\left[G_{1} \cdot x^{2}+G_{2} \cdot z^{2}-2 N \cdot x z\right] \\
& -\frac{1}{2} \beta_{0}^{2} \cdot\left[F_{x x} \cdot x^{2}+2 F_{x z} \cdot x z+F_{z z} \cdot z^{2}\right] .
\end{aligned}
$$


By eliminating the quantities $p_{x}$ and $p_{z}$ from the resulting canonical equations

$$
\begin{aligned}
x^{\prime} & =\frac{1}{\beta_{0}^{2}}\left[p_{x}+\beta_{0}^{2} \cdot H \cdot z\right] ; \\
p_{x}^{\prime} & =K_{x} \cdot p_{\sigma}+\left[p_{z}-\beta_{0}^{2} \cdot H \cdot x\right] \cdot H-\beta_{0}^{2} \cdot\left[G_{1} \cdot x-N \cdot z-F_{x x} \cdot x-F_{x z} \cdot z\right] ; \\
z^{\prime} & =\frac{1}{\beta_{0}^{2}}\left[p_{z}-\beta_{0}^{2} \cdot H \cdot x\right] ; \\
p_{z}^{\prime} & =K_{z} \cdot p_{\sigma}-\left[p_{x}+\beta_{0}^{2} \cdot H \cdot z\right] \cdot H-\beta_{0}^{2} \cdot\left[G_{2} \cdot z-N \cdot x-F_{x z} \cdot x-F_{z z} \cdot z\right] ; \\
\sigma^{\prime} & =\frac{1}{\beta_{0}^{2} \gamma_{0}^{2}} \cdot p_{\sigma}-\left(K_{x} \cdot x+K_{z} \cdot z\right) ; \\
p_{\sigma}^{\prime} & =\frac{e V(s)}{E_{0}} \cdot k \cdot \frac{2 \pi}{L} \cdot \cos \varphi \cdot \sigma+F_{\sigma}(s) \cdot \sigma
\end{aligned}
$$

and putting

$$
p_{\sigma}=\eta,
$$

we recover the equations (2.1), (2.2) and (2.14) with the help of (2.6) provided the linear approximation

$$
f(\eta)=\frac{1}{\beta_{0}^{2}} \cdot \eta
$$

is valid.

Since the equations of motion are linear they can be solved in the form

$$
\vec{y}(s)=\underline{M}\left(s, s_{0}\right) \vec{y}\left(s_{0}\right)
$$

with

$$
\vec{y}=\left(\begin{array}{c}
x \\
p_{x} \\
z \\
p_{z} \\
\sigma \\
p_{\sigma}
\end{array}\right)
$$

Because the variables $x, p_{x}, z, p_{z}, \sigma, p_{\sigma}$ are canonical, the transfer matrix $\underline{M}\left(s, s_{0}\right)$ is symplectic $[1]$ :

$$
\underline{M}^{T}\left(s, s_{0}\right) \cdot \underline{S} \cdot \underline{M}\left(s, s_{0}\right)=\underline{S}
$$

where

$$
\underline{S}=\left(\begin{array}{rrrrrr}
0 & -1 & 0 & 0 & 0 & 0 \\
1 & 0 & 0 & 0 & 0 & 0 \\
0 & 0 & 0 & -1 & 0 & 0 \\
0 & 0 & 1 & 0 & 0 & 0 \\
0 & 0 & 0 & 0 & 0 & -1 \\
0 & 0 & 0 & 0 & 1 & 0
\end{array}\right)
$$

In order to construct the matrix $\underline{M}\left(s, s_{0}\right)$, the quantities $F_{x x}, F_{x z}, F_{z x}, F_{z z}$ and $F_{\sigma}$ of (2.16) which describe the self induced space charge forces $F_{x}^{\text {self }}, F_{z}^{\text {self }}$ and $F_{\sigma}^{\text {self }}$ must be known. This is the topic of the next chapter. 


\section{The Beam Envelopes}

\subsection{The six dimensional ellipsoid in $x-p_{x}-z-p_{z}-\sigma-p_{\sigma}$ space}

To obtain the space charge forces we must know the particle distribution.

We will assume that at the start point, $s_{0}$, the ensemble is distributed on the surface of a six dimensional ellipsoid in $x-p_{x}-z-p_{z}-\sigma-p_{\sigma}$ space of the form

$$
\begin{aligned}
\vec{y}\left(s_{0} ; \varphi, \chi, \delta_{I}, \delta_{I I}, \delta_{I I I}\right)= & \cos \varphi \cdot \cos \chi \cdot\left[\vec{y}_{1}\left(s_{0}\right) \cdot \cos \delta_{I}+\vec{y}_{2}\left(s_{0}\right) \cdot \sin \delta_{I}\right]+ \\
& \cos \varphi \cdot \sin \chi \cdot\left[\vec{y}_{3}\left(s_{0}\right) \cdot \cos \delta_{I I}+\vec{y}_{4}\left(s_{0}\right) \cdot \sin \delta_{I I}\right]+ \\
& \sin \varphi \cdot\left[\vec{y}_{5}\left(s_{0}\right) \cdot \cos \delta_{I I I}+\vec{y}_{6}\left(s_{0}\right) \cdot \sin \delta_{I I I}\right]
\end{aligned}
$$

This ellipsoid can be spanned by six linearly independent vectors

$$
\vec{y}_{k}=\left(\begin{array}{c}
y_{k 1} \\
y_{k 2} \\
y_{k 3} \\
y_{k 4} \\
y_{k 5} \\
y_{k 6}
\end{array}\right) ; \quad(k=1,2,3,4,5,6)
$$

which are defined by the starting shape of the ellipsoid.

The corresponding vector $\vec{y}$ at position, $\mathrm{s}$, is given by

$$
\begin{aligned}
\vec{y}\left(s ; \varphi, \chi, \delta_{I}, \delta_{I I}, \delta_{I I I}\right)= & \cos \varphi \cdot \cos \chi \cdot\left[\vec{y}_{1}(s) \cdot \cos \delta_{I}+\vec{y}_{2}(s) \cdot \sin \delta_{I}\right]+ \\
& \cos \varphi \cdot \sin \chi \cdot\left[\vec{y}_{3}(s) \cdot \cos \delta_{I I}+\vec{y}_{4}(s) \cdot \sin \delta_{I I}\right]+ \\
& \sin \varphi \cdot\left[\vec{y}_{5}(s) \cdot \cos \delta_{I I I}+\vec{y}_{6}(s) \cdot \sin \delta_{I I I}\right]
\end{aligned}
$$

where

$$
\vec{y}_{k}(s)=\underline{M}\left(s, s_{0}\right) \vec{y}_{k}\left(s_{0}\right) ;(k=1,2,3,4,5,6) .
$$

Thus the ellipsoid remains an ellipsoid.

The beam envelopes can then be obtained by projecting the ellipsoid of eqn. (3.3) on the individual phase planes.

\subsection{The projections of the six dimensional ellipsoid}

To define the projections we first of all write the ellipsoid (3.3) in component form:

$$
\begin{aligned}
x\left(s ; \varphi, \chi, \delta_{I}, \delta_{I I}, \delta_{I I I}\right)= & \cos \varphi \cdot \cos \chi \cdot\left[y_{11}(s) \cdot \cos \delta_{I}+y_{21}(s) \cdot \sin \delta_{I}\right]+ \\
& \cos \varphi \cdot \sin \chi \cdot\left[y_{31}(s) \cdot \cos \delta_{I I}+y_{41}(s) \cdot \sin \delta_{I I}\right]+ \\
& \sin \varphi \cdot\left[y_{51}(s) \cdot \cos \delta_{I I I}+y_{61}(s) \cdot \sin \delta_{I I I}\right] ; \\
p_{x}\left(s ; \varphi, \chi, \delta_{I}, \delta_{I I}, \delta_{I I I}\right)= & \cos \varphi \cdot \cos \chi \cdot\left[y_{12}(s) \cdot \cos \delta_{I}+y_{22}(s) \cdot \sin \delta_{I}\right]+ \\
& \cos \varphi \cdot \sin \chi \cdot\left[y_{32}(s) \cdot \cos \delta_{I I}+y_{42}(s) \cdot \sin \delta_{I I}\right]+ \\
& \sin \varphi \cdot\left[y_{52}(s) \cdot \cos \delta_{I I I}+y_{62}(s) \cdot \sin \delta_{I I I}\right] ;
\end{aligned}
$$




$$
\begin{aligned}
z\left(s ; \varphi, \chi, \delta_{I}, \delta_{I I}, \delta_{I I I}\right)= & \cos \varphi \cdot \cos \chi \cdot\left[y_{13}(s) \cdot \cos \delta_{I}+y_{23}(s) \cdot \sin \delta_{I}\right]+ \\
& \cos \varphi \cdot \sin \chi \cdot\left[y_{33}(s) \cdot \cos \delta_{I I}+y_{43}(s) \cdot \sin \delta_{I I}\right]+ \\
& \sin \varphi \cdot\left[y_{53}(s) \cdot \cos \delta_{I I I}+y_{63}(s) \cdot \sin \delta_{I I I}\right] ; \\
p_{z}\left(s ; \varphi, \chi, \delta_{I}, \delta_{I I}, \delta_{I I I}\right)= & \cos \varphi \cdot \cos \chi \cdot\left[y_{14}(s) \cdot \cos \delta_{I}+y_{24}(s) \cdot \sin \delta_{I}\right]+ \\
& \cos \varphi \cdot \sin \chi \cdot\left[y_{34}(s) \cdot \cos \delta_{I I}+y_{44}(s) \cdot \sin \delta_{I I}\right]+ \\
& \sin \varphi \cdot\left[y_{54}(s) \cdot \cos \delta_{I I I}+y_{64}(s) \cdot \sin \delta_{I I I}\right] ; \\
\sigma\left(s ; \varphi, \chi, \delta_{I}, \delta_{I I}, \delta_{I I I}\right)= & \cos \varphi \cdot \cos \chi \cdot\left[y_{15}(s) \cdot \cos \delta_{I}+y_{25}(s) \cdot \sin \delta_{I}\right]+ \\
& \cos \varphi \cdot \sin \chi \cdot\left[y_{35}(s) \cdot \cos \delta_{I I}+y_{45}(s) \cdot \sin \delta_{I I}\right]+ \\
& \sin \varphi \cdot\left[y_{55}(s) \cdot \cos \delta_{I I I}+y_{65}(s) \cdot \sin \delta_{I I I}\right] ; \\
& \cos \varphi \cdot \cos \chi \cdot\left[y_{16}(s) \cdot \cos \delta_{I}+y_{26}(s) \cdot \sin \delta_{I}\right]+ \\
p_{\sigma}\left(s ; \varphi, \chi, \delta_{I}, \delta_{I I}, \delta_{I I I}\right)= & \cos \varphi \cdot \sin \chi \cdot\left[y_{36}(s) \cdot \cos \delta_{I I}+y_{46}(s) \cdot \sin \delta_{I I}\right]+ \\
& \sin \varphi \cdot\left[y_{56}(s) \cdot \cos \delta_{I I I}+y_{66}(s) \cdot \sin \delta_{I I I}\right]
\end{aligned}
$$

The computation of the single projections is then similar to that in Ref. 1 in which the functional relationship between pairs of components was investigated.

Since the details of the method have already been given in Refs. 1 and 3 only a summary will be needed here.

\subsubsection{Projection on the $x-z$ plane.}

We first investigate the projection on the $x-z$ plane. This describes the beam cross section. We will need the maximum amplitude in the $\mathrm{x}$ and $\mathrm{z}$ directions.

a) Maximum oscillation amplitude in the $\mathrm{x}$ direction:

Using the relation

$$
\operatorname{Max}_{(\varphi)}\{A \cdot \cos \varphi+B \cdot \sin \varphi\}=\sqrt{A^{2}+B^{2}}
$$

and eqn. (3.5a), the largest possible $\mathrm{x}$ amplitude is

$$
\operatorname{Max}_{\left(\varphi, \chi, \delta_{I}, \delta_{I I}, \delta_{I I I}\right)} x\left(s ; \varphi, \chi, \delta_{I}, \delta_{I I}, \delta_{I I I}\right)=\sqrt{y_{11}^{2}+y_{21}^{2}+y_{31}^{2}+y_{41}^{2}+y_{51}^{2}+y_{61}^{2}}=E_{x}(s)
$$

This occurs for the values:

$$
\begin{gathered}
\cos \delta_{I}=\frac{y_{11}}{\sqrt{y_{11}^{2}+y_{21}^{2}}} ; \quad \sin \delta_{I}=\frac{y_{21}}{\sqrt{y_{11}^{2}+y_{21}^{2}}} ; \\
\cos \delta_{I I}=\frac{y_{31}}{\sqrt{y_{31}^{2}+y_{41}^{2}}} ; \quad \sin \delta_{I I}=\frac{y_{41}}{\sqrt{y_{31}^{2}+y_{41}^{2}}} ; \\
\cos \delta_{I I I}=\frac{y_{51}}{\sqrt{y_{51}^{2}+y_{61}^{2}}} ; \quad \sin \delta_{I I I}=\frac{y_{61}}{\sqrt{y_{51}^{2}+y_{61}^{2}}}
\end{gathered}
$$




$$
\begin{gathered}
\cos \chi=\frac{\sqrt{y_{11}^{2}+y_{21}^{2}}}{\sqrt{y_{11}^{2}+y_{21}^{2}+y_{31}^{2}+y_{41}^{2}}} ; \\
\sin \chi=\frac{\sqrt{y_{31}^{2}+y_{41}^{2}}}{\sqrt{y_{11}^{2}+y_{21}^{2}+y_{31}^{2}+y_{41}^{2}}} ; \\
\cos \varphi=\frac{\sqrt{y_{11}^{2}+y_{21}^{2}+y_{31}^{2}+y_{41}^{2}}}{\sqrt{y_{11}^{2}+y_{21}^{2}+y_{31}^{2}+y_{41}^{2}+y_{51}^{2}+y_{61}^{2}}} ; \\
\sin \varphi=\frac{\sqrt{y_{51}^{2}+y_{61}^{2}}}{\sqrt{y_{11}^{2}+y_{21}^{2}+y_{31}^{2}+y_{41}^{2}+y_{51}^{2}+y_{61}^{2}}} .
\end{gathered}
$$

The corresponding $z$ coordinate is given by eqn. (3.5c) together with eqn. (3.9):

$$
G_{x}=\frac{1}{E_{x}(s)} \cdot\left\{y_{11} \cdot y_{13}+y_{21} \cdot y_{23}+y_{31} \cdot y_{33}+y_{41} \cdot y_{43}+y_{51} \cdot y_{53}+y_{61} \cdot y_{63}\right\} \text {. }
$$

b) Maximum oscillation amplitude in the $\mathrm{z}$ direction:

Correspondingly, the maximum amplitude in the $\mathrm{z}$-direction is obtained from eqn. (3.5c):

$$
\operatorname{Max}_{\left(\varphi, \chi, \delta_{I}, \delta_{I I}, \delta_{I I I}\right)} z\left(s ; \varphi, \chi, \delta_{I}, \delta_{I I}, \delta_{I I I}\right)=\sqrt{y_{13}^{2}+y_{23}^{2}+y_{33}^{2}+y_{43}^{2}+y_{53}^{2}+y_{63}^{2}}=E_{z}(s)
$$

The accompanying $\mathrm{x}$-coordinate is then:

$$
G_{z}=\frac{1}{E_{z}(s)} \cdot\left\{y_{11} \cdot y_{13}+y_{21} \cdot y_{23}+y_{31} \cdot y_{33}+y_{41} \cdot y_{43}+y_{51} \cdot y_{53}+y_{61} \cdot y_{63}\right\} \text {. }
$$

Thus

$$
E_{x} \cdot G_{x}=E_{z} \cdot G_{z}
$$

d) The boundary curve of the beam cross section.

The projections of the ellipsoid (3.5) are ellipses, and these are described by the three independent quantities $E_{x}, G_{x}, E_{z}$. The parameter $G_{z}$ depends on the other three (see eqn. (3.11)). In terms of $E_{x}, G_{x}, E_{z}$, the ellipse can be written as:

$$
E_{z}^{2} \cdot x^{2}-2 E_{x} G_{x} \cdot x z+E_{x}^{2} \cdot z^{2}=\epsilon_{x z}^{2}
$$

with

$$
\epsilon_{x z}=E_{x} \cdot \sqrt{E_{z}^{2}-G_{x}^{2}}
$$

and where $\pi \epsilon_{x z}$ is the area of the ellipse.

The half axes $E_{1}$ and $E_{2}$ of the elliptical beam cross section are:

$$
E_{1,2}=\frac{1}{2}\left\{\left[E_{x}^{2}+E_{z}^{2}\right] \pm \sqrt{\left[E_{x}^{2}-E_{z}^{2}\right]^{2}+4 E_{x}^{2} G_{x}^{2}}\right\}
$$


and the twist angle $\theta$ of the beam is given by:

$$
\tan 2 \theta=\frac{2 E_{x} G_{x}}{E_{x}^{2}-E_{z}^{2}} .
$$

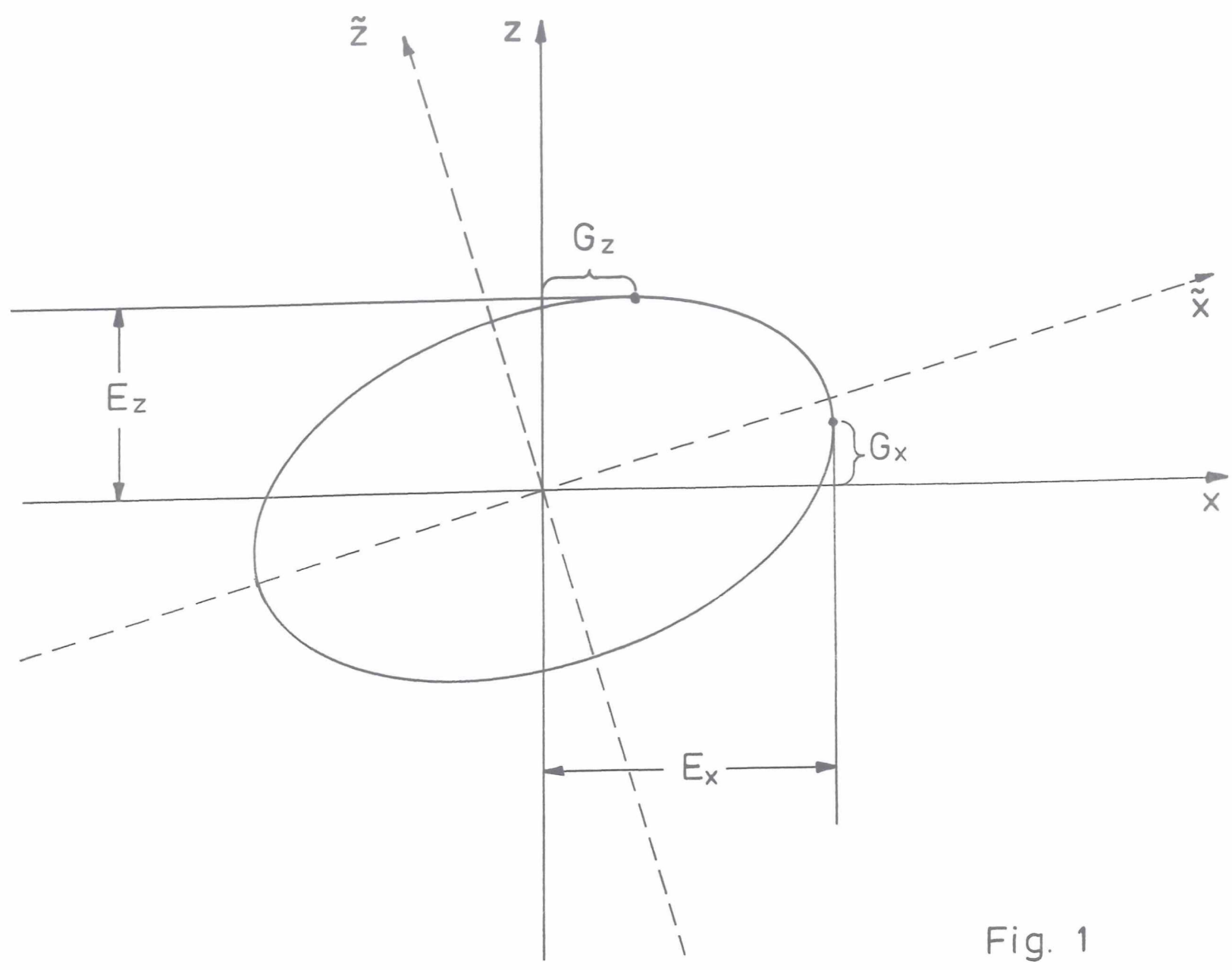

\subsubsection{Projection on the $x-\sigma$ plane.}

To find the projection of the ellipsoid (3.3) onto the $x-\sigma$ plane we need equations (3.5a) and (3.5e). Since these have the same general form as eqns. (3.5a) and (3.5c), we can obtain the projections using exactly the same methods as in the previous section.

The boundary curve of the elliptical projection on the $x-\sigma$ plane is:

$$
E_{x}^{2} \cdot \sigma^{2}-2 E_{\sigma} G_{\sigma}^{(x)} \cdot \sigma x+E_{\sigma}^{2} \cdot x^{2}=\epsilon_{\sigma x}^{2}
$$

with

$$
E_{\sigma}=\sqrt{y_{15}^{2}+y_{25}^{2}+y_{35}^{2}+y_{45}^{2}+y_{55}^{2}+y_{65}^{2}} ;
$$




$$
\begin{aligned}
G_{\sigma}^{(x)} & =\frac{1}{E_{\sigma}} \cdot\left\{y_{11} \cdot y_{15}+y_{21} \cdot y_{25}+y_{31} \cdot y_{35}+y_{41} \cdot y_{45}+y_{51} \cdot y_{55}+y_{61} \cdot y_{65}\right\} \\
\epsilon_{\sigma x} & =E_{\sigma} \cdot \sqrt{E_{x}^{2}-\left(G_{\sigma}^{(x)}\right)^{2}}
\end{aligned}
$$

The meaning of $E_{\sigma}$ and $G_{\sigma}^{(x)}$ is explained by Fig.2. $\pi \epsilon_{\sigma x}$ is the area of the ellipse (3.15).

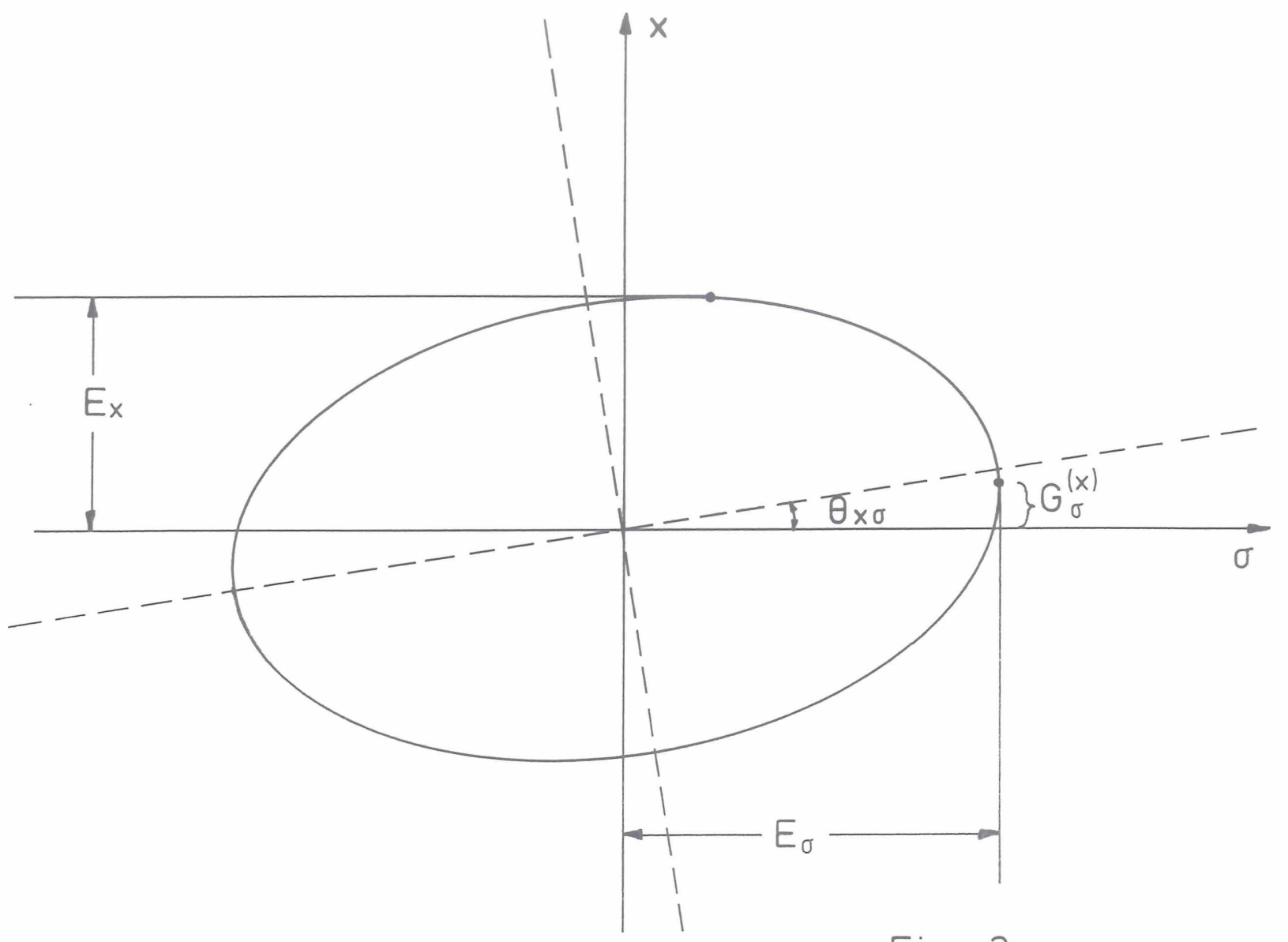

Fig. 2

\subsubsection{Projection on the $z-\sigma$ plane.}

Finally, the projection of the ellipsoid on the $z-\sigma$ plane (see Fig.3) has the boundary curve:

$$
E_{z}^{2} \cdot \sigma^{2}-2 E_{\sigma} G_{\sigma}^{(z)} \cdot \sigma z+E_{\sigma}^{2} \cdot z^{2}=\epsilon_{\sigma z}^{2}
$$

with

$$
\begin{aligned}
G_{\sigma}^{(z)} & =\frac{1}{E_{\sigma}} \cdot\left\{y_{13} \cdot y_{15}+y_{23} \cdot y_{25}+y_{33} \cdot y_{35}+y_{43} \cdot y_{45}+y_{53} \cdot y_{55}+y_{63} \cdot y_{65}\right\} \\
\epsilon_{\sigma z} & =E_{\sigma} \cdot \sqrt{E_{z}^{2}-\left(G_{\sigma}^{(z)}\right)^{2}}
\end{aligned}
$$

$\left(\pi \epsilon_{\sigma z}\right.$ is the area of the ellipse (3.17)). 
Now that we have the projections of the ellipsoid on all three planes we can calculate the space charge forces. We assume that the particle distribution is uniform in the $\mathrm{x}$ - $\mathrm{z}$-s space.

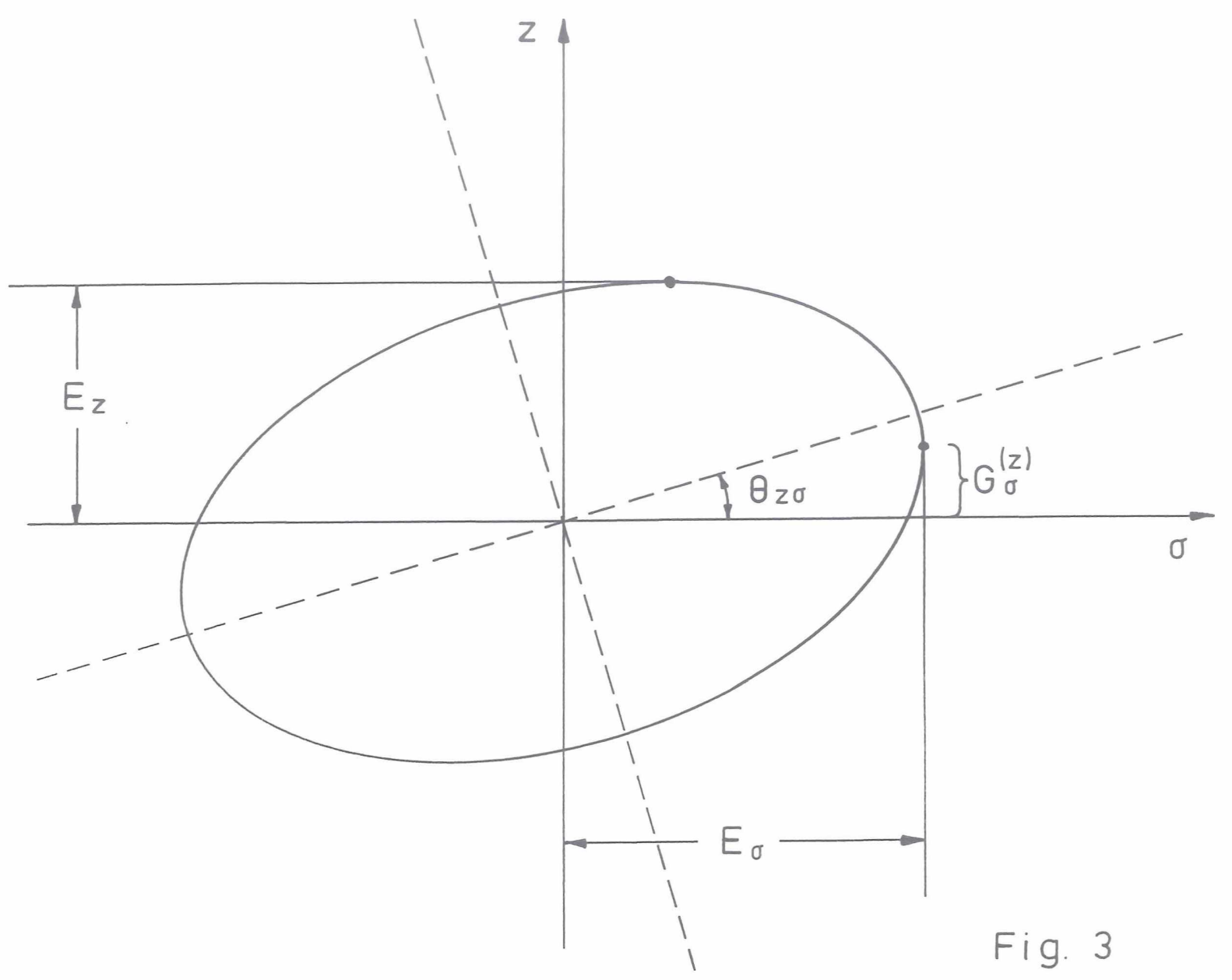

\section{Calculation of Space Charge Forces}

In calculating the space charge forces, we assume that the synchro-betatron coupling is small and we therefore ignore the twist angles $\theta_{x \sigma}$ and $\theta_{z \sigma}$ of the bunch with respect to the $\sigma$ axis (Fig. 2, 3).

Since (see chapter 2) the variable $\sigma$ describes the distance of a particle from the centre of the bunch, then $E_{\sigma}$ describes the half length of the bunch in the laboratory system. $E_{1}$ and $E_{2}$ describe the transverse bunch extensions.

The whole bunch can now be represented by a three dimensional ellipsoid which, in a rotated $(\tilde{x}, \tilde{z}, \sigma)$ coordinate system (see Fig.1), can be described by the equation

$$
\frac{\tilde{x}^{2}}{E_{1}^{2}}+\frac{\tilde{z}^{2}}{E_{2}^{2}}+\frac{\sigma^{2}}{E_{\sigma}^{2}}=1
$$


in the laboratory system and by the equation

$$
\frac{\tilde{x}^{2}}{E_{1}^{2}}+\frac{\tilde{z}^{2}}{E_{2}^{2}}+\frac{\bar{\sigma}^{2}}{\left(\gamma_{0} \cdot E_{\sigma}\right)^{2}}=1
$$

in the rest system of the bunch $\left(\bar{\sigma}=\gamma_{0} \cdot \sigma\right.$ describes the longitudinal coordinate in the rest system) .

Now for a uniform charge distribution in the (rest system) ellipsoid

$$
\frac{\xi^{2}}{a^{2}}+\frac{\eta^{2}}{b^{2}}+\frac{\zeta^{2}}{c^{2}}=1
$$

the potential inside is given by $[4]$

$$
U=-A \cdot \xi^{2}-B \cdot \eta^{2}-C \cdot \zeta^{2}+D
$$

with

$$
\begin{aligned}
& A=\pi a b c \cdot \rho \cdot \int_{0}^{\infty} \frac{d \tau}{\left(a^{2}+\tau\right) \cdot \sqrt{\varphi(\tau)}} ; \\
& B= \pi a b c \cdot \rho \cdot \int_{0}^{\infty} \frac{d \tau}{\left(b^{2}+\tau\right) \cdot \sqrt{\varphi(\tau)}} ; \\
& C= \pi a b c \cdot \rho \cdot \int_{0}^{\infty} \frac{d \tau}{\left(c^{2}+\tau\right) \cdot \sqrt{\varphi(\tau)}} ; \\
& D= \pi a b c \cdot \rho \cdot \int_{0}^{\infty} \frac{d \tau}{\sqrt{\varphi(\tau)}} ; \\
& \varphi(\tau)=\left(a^{2}+\tau\right) \cdot\left(b^{2}+\tau\right) \cdot\left(c^{2}+\tau\right) ; \\
& \quad(\rho=\text { charge density }) .
\end{aligned}
$$

The total charge in the bunch is

$$
Q=\frac{4 \pi}{3} a b c \cdot \rho .
$$

Thus comparing (4.2) and (4.3) the space charge force in the rest system is

$$
\begin{aligned}
& F_{\tilde{x}}^{(0)}=\frac{3}{2} e Q \cdot I_{1} \cdot \tilde{x} ; \\
& F_{\tilde{z}}^{(0)}=\frac{3}{2} e Q \cdot I_{2} \cdot \tilde{z} \\
& F_{s}^{(0)}=\frac{3}{2} e Q \cdot I_{3} \cdot \bar{\sigma}
\end{aligned}
$$

with

$$
\begin{aligned}
I_{1} & =\int_{0}^{\infty} \frac{d \tau}{\left(E_{1}^{2}+\tau\right) \cdot \sqrt{\psi(\tau)}} ; \\
I_{2} & =\int_{0}^{\infty} \frac{d \tau}{\left(E_{2}^{2}+\tau\right) \cdot \sqrt{\psi(\tau)}} ; \\
I_{3} & =\int_{0}^{\infty} \frac{d \tau}{\left(\gamma_{0}^{2} \cdot E_{\sigma}^{2}+\tau\right) \cdot \sqrt{\psi(\tau)}} ; \\
\psi(\tau) & =\left(E_{1}^{2}+\tau\right) \cdot\left(E_{2}^{2}+\tau\right) \cdot\left(\gamma_{0}^{2} E_{\sigma}^{2}+\tau\right) .
\end{aligned}
$$


The terms $I_{1}, I_{2}, I_{3}$ can be expressed in terms of elliptical integrals of the second kind.

As shown in Appendix $A$, if

$$
\gamma_{0} E_{\sigma} \gg E_{1}, E_{2}
$$

$I_{1}, I_{2}, I_{3}$ can be calculated approximately by analytical means.

We now Lorentz transform to the laboratory frame to obtain the forces

$$
\begin{aligned}
& F_{\tilde{x}}=\frac{1}{\gamma_{0}} \cdot F_{\tilde{x}}^{(0)} ; \\
& F_{\tilde{z}}=\frac{1}{\gamma_{0}} \cdot F_{\tilde{z}}^{(0)} ; \\
& F_{s}=F_{s}^{(0)}
\end{aligned}
$$

so that the space charge forces in the laboratory system are

$$
\begin{aligned}
F_{\tilde{x}}^{\text {self }} & =\frac{1}{\gamma_{0}} \cdot \frac{3}{2} e Q \cdot I_{1} \cdot \tilde{x} ; \\
F_{\tilde{z}}^{\text {self }} & =\frac{1}{\gamma_{0}} \cdot \frac{3}{2} e Q \cdot I_{2} \cdot \tilde{z} ; \\
F_{s}^{\text {self }} & =\frac{3}{2} e Q \cdot I_{3} \cdot \gamma_{0} \cdot \sigma,
\end{aligned}
$$

where in $(4.7 \mathrm{c})$ we use the relation (Lorentz contraction):

$$
\bar{\sigma}=\gamma_{0} \cdot \sigma
$$

The components of space charge force in $\mathrm{x}$ and $\mathrm{z}$ directions are

$$
\begin{aligned}
F_{x}^{\text {self }} & =\cos \theta \cdot F_{\tilde{x}}-\sin \theta \cdot F_{\tilde{z}} \\
& =\frac{1}{\gamma_{0}} \cdot \frac{3}{2} e Q \cdot\left[x \cdot\left(I_{1} \cos ^{2} \theta+I_{2} \sin ^{2} \theta\right)+z \cdot \sin \theta \cos \theta \cdot\left(I_{1}-I_{2}\right)\right] \\
F_{x}^{\text {self }} & =\sin \theta \cdot F_{\tilde{x}}+\cos \theta \cdot F_{\tilde{z}} \\
& =\frac{1}{\gamma_{0}} \cdot \frac{3}{2} e Q \cdot\left[x \cdot \sin \theta \cos \theta \cdot\left(I_{1}-I_{2}\right)+z \cdot\left(I_{1} \sin ^{2} \theta+I_{2} \cos ^{2} \theta\right)\right] .
\end{aligned}
$$

By comparing eqn. (4.7c) and eqns. (4.8a,b) with (2.11) and (2.5a,b) we finally obtain the coefficients $F_{x x}, F_{x z}, F_{z x}, F_{z z}$ and $F_{\sigma}$ :

$$
\begin{aligned}
F_{x x} & \left.=\frac{3}{2} e Q \cdot \frac{1}{\gamma_{0}^{2} \cdot m_{0} v_{0}^{2}} \cdot\left[I_{1} \cos ^{2} \theta+I_{2} \sin ^{2} \theta\right)\right] \\
F_{z z} & \left.=\frac{3}{2} e Q \cdot \frac{1}{\gamma_{0}^{2} \cdot m_{0} v_{0}^{2}} \cdot\left[I_{1} \sin ^{2} \theta+I_{2} \cos ^{2} \theta\right)\right] ; \\
F_{x z} & =\frac{3}{2} e Q \cdot \frac{1}{\gamma_{0}^{2} \cdot m_{0} v_{0}^{2}} \cdot \sin \theta \cos \theta \cdot\left(I_{1}-I_{2}\right) \\
& =F_{z x} ; \\
F_{\sigma} & =\frac{3}{2} e Q \cdot \frac{\gamma_{0}}{E_{0}} \cdot I_{3} .
\end{aligned}
$$


The angle $\theta$ is defined by eqn. (3.14) and the quantities $I_{1}, I_{2}$ and $I_{3}$ by eqn. (4.6).

In particular, we see that eqn. (4.9c) reproduces eqn. (2.6) which was used to derive the Hamiltonian (2.15).

Equation (4.9) can now be used together with eqn. (2.16) (and with the help of (3.6), (3.8), (3.9), (3.16a), (3.13) and (3.14)) to obtain explicit forms for the (canonical) equations of motion under the influence of both external and space charge effects.

\section{Remark:}

In eqn. (4.8) the effects of orbit curvature described in Ref. 5 are not included but the linear part of these additional forces could be easily incorporated. Linear wakefield effects could be taken into account in the same way.

\section{Solution of the Equations of Motion}

The solution of these equations will be obtained in transport matrix form. We write

$$
\frac{d}{d s} \vec{y}=\underline{A}(s) \cdot \vec{y}
$$

with

$$
\begin{aligned}
& A_{12}=\frac{1}{\beta_{0}^{2}} \\
& A_{13}=+H ; \\
& A_{21}=-\beta_{0}^{2} \cdot\left[G_{1}-F_{x x}+H^{2}\right] ; \\
& A_{23}=+\beta_{0}^{2} \cdot\left[N+F_{x z}\right] ; \\
& A_{24}=+H ; \\
& A_{26}=K_{x} ; \\
& A_{31}=-H ; \\
& A_{34}=\frac{1}{\beta_{0}^{2}} ; \\
& A_{41}=+\beta_{0}^{2} \cdot\left[N+F_{x z}\right] ; \\
& A_{42}=-H ; \\
& A_{43}=-\beta_{0}^{2} \cdot\left[G_{2}-F_{z z}+H^{2}\right] ; \\
& A_{46}=K_{z} ; \\
& A_{51}=-K_{x} ; \\
& A_{53}=-K_{z} ; \\
& A_{56}=\frac{1}{\beta_{0}^{2} \cdot \gamma_{0}^{2}} ; \\
& A_{65}=\frac{e V(s)}{E_{0}} \cdot k \cdot \frac{2 \pi}{L} \cdot \cos \varphi+F_{\sigma} ; \\
& A_{i k}=0 \text { otherwise }
\end{aligned}
$$

We solve eqn. (5.1) using thin lens approximation [1]. 
Using notation similar to that of Ref. 1, we obtain for the transfer matrix

$$
\underline{M}(s+\Delta s, s)=\underline{M}_{D}\left(s+\Delta s, s+\frac{\Delta s}{2}\right) \cdot[\underline{1}+\underline{C}(s) \cdot \Delta s] \cdot \underline{R}(\Delta \Theta) \cdot \underline{M}_{D}\left(s+\frac{\Delta s}{2}, s\right)
$$

with

$$
\begin{aligned}
\underline{C}(s) & =\underline{A}(s)-\underline{D}-\underline{F} ; \\
\underline{D} & =\underline{1} \bar{\beta}_{0}^{2} \cdot\left(\begin{array}{rrrrrrr}
0 & 1 & 0 & 0 & 0 & 0 \\
0 & 0 & 0 & 0 & 0 & 0 \\
0 & 0 & 0 & 1 & 0 & 0 \\
0 & 0 & 0 & 0 & 0 & 0 \\
0 & 0 & 0 & 0 & 0 & 1 / \gamma_{0}^{2} \\
0 & 0 & 0 & 0 & 0 & 0
\end{array}\right) ; \\
\underline{F} & =H \cdot\left(\begin{array}{rrrrrr}
0 & 0 & 1 & 0 & 0 & 0 \\
0 & 0 & 0 & 1 & 0 & 0 \\
-1 & 0 & 0 & 0 & 0 & 0 \\
0 & -1 & 0 & 0 & 0 & 0 \\
0 & 0 & 0 & 0 & 0 & 0 \\
0 & 0 & 0 & 0 & 0 & 0
\end{array}\right) ; \\
\underline{M}_{D}(s+l, s) & =\underline{1}+l \cdot \underline{D} ; \quad r r i f t
\end{aligned}
$$

(transfer matrix for a simple drift space of length 1);

$$
\underline{R}(\Delta \Theta)=\left(\begin{array}{rrrrrr}
\cos \Delta \Theta & 0 & +\sin \Delta \Theta & 0 & 0 & 0 \\
0 & \cos \Delta \Theta & 0 & +\sin \Delta \Theta & 0 & 0 \\
-\sin \Delta \Theta & 0 & \cos \Delta \Theta & 0 & 0 & 0 \\
0 & -\sin \Delta \Theta & 0 & \cos \Delta \Theta & 0 & 0 \\
0 & 0 & 0 & 0 & 1 & 0 \\
0 & 0 & 0 & 0 & 0 & 1
\end{array}\right) ;
$$

As in Ref. 1, this thin lens form is symplectic and the matrix $\underline{A}$ depends on the shape of the bunch. The latter depends on the generating orbit vectors $\vec{y}_{k}(k=1,2,3,4,5,6)$ which change during the motion of the bunch according to the equation [1]

$$
\vec{y}_{k}(s+\Delta s)=\underline{M}(s+\Delta s, s) \cdot \vec{y}_{k}(s) .
$$

Periodic solutions must be obtained by self consistent iteration [1].

Finally, we point out that the 6 equations (5.5) for the generating vectors $\vec{y}_{k}(k=1-6)$ can be handled in a compact way by introducing the "bunch-shape matrix"

$$
\underline{B}=\left(\overrightarrow{y_{1}}, \overrightarrow{y_{2}}, \overrightarrow{y_{3}}, \overrightarrow{y_{4}}, \overrightarrow{y_{5}}, \overrightarrow{y_{6}}\right)
$$

so that

$$
\underline{B}(s+\Delta s, s)=\underline{\hat{M}}(s+\Delta s, s) \cdot \underline{B}(s) .
$$

Acceleration by a cavity field is described in Appendix B. 


\section{Summary}

We have investigated the influence of longitudinal and transverse space charge forces on the motion of charged particles in storage rings and transport systems by a simultanous treatment of synchrotron and betatron oscillations.

The motion is described in terms of the fully six-dimensional formalism with the canonical variables $x, p_{x}, z, p_{z}, \sigma=s-v_{0} \cdot t, p_{\sigma}=\Delta E / E_{0}$.

In order to describe the bunch we have introduced a 6-dimensional ellipsoid in the $x-$ $p_{x}-z-p_{z}-\sigma-p_{\sigma}$ space represented by the "bunch-shape matrix", $\underline{\mathrm{B}}(\mathrm{s})$, which contains as columns, six independent orbit vectors. As in Ref. 1, this matrix $\underline{B}(\mathrm{~s})$ contains complete information about the configuration of the bunch at the point $\mathrm{s}$ and can be obtained by matrix multiplication with the transfer matrix $\underline{\mathrm{M}}$.

In thin lens approximation the matrix takes a simple form which can be conveniently coded for computer.

The equations so derived could be used for studying beam transport in DESY III .

\section{Acknowledgements}

We wish to thank Dr. D. P. Barber for many stimulating discussions and guidance, for helping to translate the text and for careful reading of the manuscript. We also thank Prof. Dr. B. Wiik for continued encouragement and Dr. Y. H. Chin, Dr. A. Piwinski and Dr. F. Willeke for helpful and interesting discussions.

\section{Appendix A: Calculation of the Space Charge Integrals $I_{1}, I_{2}, I_{3}$}

In order to calculate the space charge integrals $I_{1}, I_{2}, I_{3}$ of eqn. (4.6) we assume that

$$
E_{3}=\gamma_{0} E_{\sigma} \gg E_{1}, E_{2} \text {. }
$$

In this case, for $I_{1}$ we may write (approximately):

$$
I_{1} \approx \frac{1}{E_{3}} \cdot \int_{0}^{\infty} \frac{d \tau}{\left(E_{1}^{2}+\tau\right) \cdot \sqrt{\left(E_{1}^{2}+\tau\right) \cdot\left(E_{2}^{2}+\tau\right)}} .
$$

With the substitution

$$
t^{2}=\tau+E_{2}^{2}
$$

we get

$$
\begin{aligned}
I_{1} & \approx \frac{2}{E_{3}} \cdot \int_{E_{2}}^{\infty} \frac{d t}{\sqrt{t^{2}+E_{1}^{2}-E_{2}^{2}}} \\
& =\frac{2}{E_{3}} \cdot \frac{1}{E_{1}^{2}-E_{2}^{2}} \cdot\left[\frac{t}{\sqrt{t^{2}+E_{1}^{2}-E_{2}^{2}}}\right]_{E_{2}}^{\infty} \\
& =\frac{2}{E_{3}} \cdot \frac{1}{E_{1}\left(E_{1}+E_{2}\right)} .
\end{aligned}
$$


$I_{2}$ can be obtained in the same way:

$$
I_{2} \approx \frac{2}{E_{3}} \cdot \frac{1}{E_{2}\left(E_{1}+E_{2}\right)} .
$$

Finally, also using assumption (A.1):

$$
I_{3} \approx \frac{1}{E_{3}^{3}} \cdot \int_{0}^{E_{3}} \frac{d \tau}{\sqrt{\left(E_{1}^{2}+\tau\right) \cdot\left(E_{2}^{2}+\tau\right)}}
$$

or with the substitution (A.3):

$$
\begin{aligned}
I_{3} & \approx \frac{2}{E_{3}^{3}} \cdot \int_{E_{2}}^{E_{3}} \frac{d t}{\sqrt{t^{2}+E_{1}^{2}-E_{2}^{2}}} \\
& =\frac{2}{E_{3}^{3}} \cdot\left[\ln \left(t+\sqrt{t^{2}+E_{1}^{2}-E_{2}^{2}}\right)\right]_{E_{2}}^{E_{3}} \\
& =\frac{2}{E_{3}^{3}} \cdot \ln \frac{E_{3}+\sqrt{E_{3}^{2}+E_{1}^{2}-E_{2}^{2}}}{E_{1}+E_{2}} .
\end{aligned}
$$

\section{Appendix B: The Acceleration Process}

In the solution of the equations of motion we assumed that the average energy $E_{0}$ remained constant i.e. that the cavity phase was either set at 0 or $\pi$ so that no acceleration took place (see eqn. (2.10).

To describe the acceleration process we now consider the case where

$$
\sin \varphi \neq 0, \pi .
$$

In linear approximation, the cavity field $\varepsilon_{\text {Cavity }}$ varies as

$$
\varepsilon_{\text {Cavity }}=V(s) \sin \varphi+V(s) \cos \varphi \cdot k \cdot \frac{2 \pi}{L} \cdot \sigma,
$$

and if the cavity is point like at $s=s_{0}$ :

$$
V(s)=\hat{V} \cdot \delta\left(s-s_{0}\right)
$$

the energy gain is

$$
E\left(s+s_{0}\right)-E\left(s-s_{0}\right)=e \hat{V} \sin \varphi+e \hat{V} \cos \varphi \cdot k \cdot \frac{2 \pi}{L} \cdot \sigma\left(s_{0}-0\right) .
$$

The average energy gain is thus

$$
\Delta E_{0}=e \hat{V} \cdot \sin \varphi,
$$

so that we can put :

$$
\begin{aligned}
E_{0}\left(s_{0}+0\right) & =E_{0}\left(s_{0}-0\right)+\Delta E_{0} ; \\
p_{0}\left(s_{0}+0\right) & =\sqrt{\frac{1}{c^{2}} \cdot E_{0}^{2}\left(s_{0}+0\right)-m_{0} c^{2}} \\
v_{0}\left(s_{0}+0\right) & =\frac{c^{2} \cdot p_{0}\left(s_{0}+0\right)}{E_{0}\left(s_{0}+0\right)} .
\end{aligned}
$$


Writing the variable

$$
\sigma(s)=s-v_{0} \cdot t(s)
$$

in the form

$$
\sigma(s)=v_{0}(s) \cdot\left[t_{0}(s)-t(s)\right]
$$

$\left(t_{0}(s)\right.$ is the time for the synchronous particle) and recalling that $t_{0}(s)$ and $t(s)$ are continous functions:

$$
\begin{aligned}
t_{0}\left(s_{0}+0\right) & =t_{0}\left(s_{0}-0\right) \\
t\left(s_{0}+0\right) & =t\left(s_{0}-0\right)
\end{aligned}
$$

we then obtain

$$
\sigma\left(s_{0}+0\right)=\frac{v_{0}\left(s_{0}+0\right)}{v_{0}\left(s_{0}-0\right)} \cdot \sigma\left(s_{0}-0\right) .
$$

Furthermore, using (B.3), (B.4) and (B.5) we find that

$$
\begin{aligned}
\eta\left(s_{0}+0\right) & =\frac{E\left(s_{0}+0\right)-E_{0}\left(s_{0}+0\right)}{E_{0}\left(s_{0}+0\right)} \\
& =\frac{1}{E_{0}\left(s_{0}+0\right)} \cdot\left[E_{0}\left(s_{0}-0\right) \cdot \eta\left(s_{0}-0\right)+e \hat{V} \cdot \cos \varphi \cdot k \cdot \frac{2 \pi}{L} \cdot \sigma\left(s_{0}-0\right) \cdot\right]
\end{aligned}
$$

For the variables $x, x^{\prime}, z, z^{\prime}$ of the transverse motions we have (see Ref. 2) :

$$
\begin{aligned}
x\left(s_{0}+0\right) & =x\left(s_{0}-0\right) \\
x^{\prime}\left(s_{0}+0\right) & =\frac{p_{0}\left(s_{0}-0\right)}{p_{0}\left(s_{0}+0\right)} \cdot x^{\prime}\left(s_{0}-0\right) ; \\
z\left(s_{0}+0\right) & =z\left(s_{0}-0\right) \\
z^{\prime}\left(s_{0}+0\right) & =\frac{p_{0}\left(s_{0}-0\right)}{p_{0}\left(s_{0}+0\right)} \cdot z^{\prime}\left(s_{0}-0\right) .
\end{aligned}
$$

Equations (B.8), (B.9) and (B.10) can now be collected together in matrix form to give

$$
\vec{y}(s+0)=\underline{M}_{\text {Cavity }}\left(s_{0}+0, s_{0}-0\right) \cdot \vec{y}(s-0)
$$

where

$$
\begin{aligned}
M_{\text {Cavity }}\left(s_{0}+0, s_{0}-0\right) & =\left(\left(M_{i k}\right)\right) ; \\
M_{11} & =1 ; \\
M_{22} & =\frac{p_{0}\left(s_{0}-0\right)}{p_{0}\left(s_{0}+0\right)} ; \\
M_{33} & =1 ; \\
M_{44} & =M_{22} ; \\
M_{55} & =\frac{v_{0}\left(s_{0}-0\right)}{v_{0}\left(s_{0}+0\right)} ; \\
M_{65} & =\frac{e \hat{V}}{E_{0}\left(s_{0}+0\right)} \cdot \cos \varphi \cdot k \cdot \frac{2 \pi}{L} ; \\
M_{66} & =\frac{E_{0}\left(s_{0}-0\right)}{E_{0}\left(s_{0}+0\right)} ; \\
M_{i k} & =0 \text { otherwise }
\end{aligned}
$$


and where $E_{0}\left(s_{0}+0\right), p_{0}\left(s_{0}+0\right), v_{0}\left(s_{0}+0\right)$ can be taken from eqns. (A.5), (A.6) and (A.7).

In particular we get for the generating vectors $\vec{y}_{k}(s)$ of the 6 -dimensional ellipsoid:

$$
\begin{gathered}
\left.\left.\vec{y}_{k}\left(s_{0}\right)+0\right)=\underline{M}_{\text {Cavity }}\left(s_{0}+0, s_{0}-0\right) \cdot \vec{y}_{k}\left(s_{0}\right)-0\right) ; \\
(k=1,2,3,4,5,6) .
\end{gathered}
$$

In the variables $\left(x, x^{\prime}, z, z^{\prime}\right)$ the transfer matrix

$$
\underline{M}_{\text {Cavity }}\left(s_{0}+0, s_{0}-0\right)
$$

is no longer symplectic and transverse damping occurs in $x, x^{\prime}, z, z^{\prime}$ space. For a symplectic treatment of the acceleration process within the framework of a non-linear theory see Ref. 6 and 7 .

The transfer matrices for the magnetic lenses remain as in chapter 5 .

\section{References}

[1] I.Borchardt, E.Karantzoulis, H.Mais, G.Ripken: "Calculation of beam envelopes in storage rings and transport systems in the presence of transverse space charge effects and coupling"; DESY 87-161.

[2] D.P.Barber, G.Ripken, F.Schmidt: "A non-linear canonical formalism for the coupled synchro-betatron motion of protons with arbitrary energy"; DESY M-87-36.

[3] G.Ripken: "Untersuchungen zur Strahlfuehrung und Stabilitaet der Teilchenbewegung in Beschleunigern und Storage-Ringen unter strenger Beruecksichtigung einer Kopplung der Betatronschwingungen"; DESY R1-70/04.

[4] O.D.Kellog: "Foundations of Potential Theory"; Springer-Verlag (1967) .

[5] The effects of orbit curvature on interparticle forces have recently been discussed in:

R.Talman: "Novel relativistic effect important in accelerators" ;

Phys.Rev.Lett. 56, p.1429 (1986).

A.Piwinski: "On the transverse forces caused by the curvature"; CERN/LEP-TH/85-43.

M.Bassetti: "Analytical formula for the centrifugal space charge effects" ; CERN/LEP$\mathrm{TH} / 86-13$.

M.Bassetti, D.Brandt: "Transverse electromagnetic forces in circular trajectory" ; CERN/LEP-TH/86-04.

[6] G.Ripken: "Non-linear canonical equations of coupled synchro- betatron motion and their solution within the framework of a non-linear 6-dimensional (symplectic) tracking program for ultra-relativistic protons"; DESY M-85-84.

[7] F.Schmidt: Phd. Theses (to be published). 